\title{
A Prospective, Multicenter, Open-Label Study of Dose Escalation Therapy in Male Patients With Nocturia Refractory to 0.2-mg Tamsulosin Monotherapy
}

\author{
Ho Song $\mathrm{Yu}^{1}$, Jeong Woo Lee ${ }^{2}$, Jihyeong $\mathrm{Yu}^{3}$, Min Chul Cho ${ }^{4}$, Sung Yong $\mathrm{Cho}^{5}$ \\ ${ }^{1}$ Department of Urology, Chonnam National University Hospital, Gwangju, Korea \\ ${ }^{2}$ Department of Urology, Dongguk University Ilsan Hospital, Dongguk University College of Medicine, Goyang, Korea \\ ${ }^{3}$ Department of Urology, Inje University Sanggye Paik Hospital, Seoul, Korea \\ ${ }^{4}$ Department of Urology, Seoul Metropolitan Government-Seoul National University Boramae Medical Center, Seoul National University College of Medicine, \\ Seoul, Korea \\ ${ }^{5}$ Department of Urology, Seoul National University Hospital, Seoul National University College of Medicine, Seoul, Korea
}

Purpose: To investigate the efficacy and safety of $0.4 \mathrm{mg}$ of tamsulosin in patients with nocturia not responding to $0.2 \mathrm{mg}$. Methods: Patients with intractable nocturia after treatment with $0.2 \mathrm{mg}$ of tamsulosin for $>1$ month were included in a multicenter, prospective, observational, single-arm study. Patients were prescribed $0.4 \mathrm{mg}$ of tamsulosin and followed up for 2 months to assess nocturnal voiding and nocturia-related bother. Changes in the mean number of nocturnal voids, the proportion of 50\% responders, 3-day frequency-volume chart parameters, and questionnaire scores were assessed.

Results: Sixty-two patients were prescribed $0.2 \mathrm{mg}$ of tamsulosin, of whom 56 were prescribed $0.4 \mathrm{mg}$ of tamsulosin. Ten patients dropped out. A single case of orthostatic hypotension was reported. The mean age was 68 years. After 1 and 2 months of taking $0.4 \mathrm{mg}$ of tamsulosin, $23.9 \%$ and $22.7 \%$ of patients demonstrated a $>50 \%$ reduction of nocturia, and $16.1 \%$ and $19.4 \%$ of patients rated the treatment as "very effective," respectively. Dose escalation to $0.4 \mathrm{mg}$ of tamsulosin, compared to $0.2 \mathrm{mg}$, did not show an additional effect on reducing nocturnal urine volume. Multivariate logistic regression analysis showed that lower serum sodium levels (odds ratio $[\mathrm{OR}], 0.41, \mathrm{P}=0.037)$ and the presence of urge incontinence $(\mathrm{OR}, 7.08, \mathrm{P}=0.036)$ were predictors of a significant improvement of nocturia in response to $0.4 \mathrm{mg}$ of tamsulosin.

Conclusions: Dose escalation may yield a significant improvement of nocturia in $>20 \%$ of patients, and may be especially helpful in patients with lower sodium levels and urge incontinence.

Keywords: Adrenergic alpha-antagonists; Nocturia; Tamsulosin

- Fund/Grant Support: This study was supported by grant from Astellas Pharma Korea Inc. (no. AKR-HA-2013-11). There are no financial or commercial interests regarding this paper.

- Research Ethics: This trial was performed in accordance with the Declaration of Helsinki, approval for this study was granted by the Institutional Review Board (IRB) of Seoul Metropolitan Government-Seoul National University Boramae Medical Center (IRB No. 16-2014-73), and the content is described according to the STROBE (Strengthening The Reporting of OBservational Studies in Epidemiology) statement.

- Conflict of Interest: This study was supported by grant from Astellas Pharma Korea Inc. (no. AKR-HA-2013-11). There are no financial or commercial interests regarding this paper. Except for that, no potential conflict of interest relevant to this article was reported.

Corresponding author: Sung Yong Cho (iD https://orcid.org/0000-0001-9271-6951 Department of Urology, Seoul National University Hospital, 101 Daehak-ro, Jongno-gu, Seoul 03080, Korea

E-mail: kmoretry@daum.net / Tel: +82-2-2072-1491 / Fax: +82-2-742-4665 Submitted: April 29, 2019 / Accepted after revision: October 20, 2019
This is an Open Access article distributed under the terms of the Creative Commons Attribution Non-Commercial License (http://creativecommons.org/licenses/by-nc/4.0/) which permits unrestricted non-commercial use, distribution, and reproduction in any medium, provided the original work is properly cited. 


\section{INTRODUCTION}

Nocturia, which is defined as waking up during the night one or more times to urinate, can seriously threaten patients' quality of life [1]. In particular, in elderly patients, nocturia increases the chances of falling and fracture, which results in a higher mortality rate [2]. Nocturia is known to have various causes, such as benign prostatic hyperplasia, bladder changes due to overactive bladder, and nocturnal polyuria. Of these, nocturnal polyuria is the most common cause of nocturia, and it has been reported that an imbalance of plasma antidiuretic hormone (arginine vasopressin) levels or natriuresis could cause nocturnal polyuria [3]. Therefore, desmopressin acetate, a synthetic analogue of arginine vasopressin, was selected as the first treatment choice for nocturia. Taking this medication before bed resulted in lower levels of urine production, which was effective for treating nocturia. However, in a previous study, $28 \%-46 \%$ of patients did not show any significant effects from this treatment; furthermore, its potential side effects include hyponatremia and loss of consciousness, which can be fatal, meaning that physicians must check patients' electrolyte levels at consultations [4]. Furthermore, desmopressin has been reported to be ineffective for treating lower urinary tract symptoms (LUTS) in patients with prostate enlargement, so the use of other medications for combination therapy needs to be considered, although doing so can increase the rate of side effect occurrence. Other medications that could be considered to improve nocturia are alpha blockers such as tamsulosin, anticholinergic agents such as solifenacin, and $5 \alpha$-reductase inhibitors such as dutasteride. Alpha blockers are the most commonly used medication to relieve LUTS in benign prostatic hyperplasia patients.

Previous studies of Asian patients with prostate enlargement who complained of LUTS with nocturia reported symptom improvement in response to a low dose $(0.2 \mathrm{mg})$ of tamsulosin $[5,6]$. If the medication does not clearly show an effect, the attending physician should consider changing the treatment plan by dose escalation to $0.4 \mathrm{mg}$, changing the drug, or add-on therapy. However, dose escalation of tamsulosin to $0.4 \mathrm{mg}$ has recently been considered the first treatment option when physicians are concerned about the side effects of other drugs or the late effects of $5 \alpha$-reductase inhibitors. Dose escalation is expected to yield additional improvement of LUTS, nocturia, and patient satisfaction $[7,8]$. Nonetheless, previous studies have rarely showed improvements of nocturia and patient satisfaction when attending physicians considered a dose escalation of tam- sulosin to $0.4 \mathrm{mg}$. Therefore, the aim of this prospective observational study was to investigate the efficacy and safety of 0.4 $\mathrm{mg}$ of tamsulosin in patients with nocturia who are not responding to $0.2 \mathrm{mg}$ of tamsulosin. The findings of this study are expected to be relevant for patients who do not want to take or change such medications as desmopressin, anticholinergic agents, beta 3 agonists, etc.

\section{MATERIALS AND METHODS}

\section{Patients}

Eligible male patients aged $>40$ years were initially prescribed $0.2 \mathrm{mg}$ of tamsulosin for $>1$ month if their International Prostate Symptom Score (IPSS) questionnaire score was 8 or higher and if their nocturia score was 2 or higher. Patients were informed of the advantages and disadvantages of taking desmopressin at a clinical consultation, and those who refused to take desmopressin first were enrolled in the study. Patients with intractable nocturia after taking $0.2 \mathrm{mg}$ of tamsulosin for $>1$ month were included in this study. Nocturia was defined as 2 or more nightly voids, as determined using a 3-day frequency-volume chart. Patients who took the same medication for $>3$ months without any dose change were included in this study. Patients were excluded from this study if they had a urinary tract infection, urolithiasis, renal function deterioration (serum creatinine $>1.4 \mathrm{mg} / \mathrm{dL}$ ), daily urine output $>2.8 \mathrm{~L}$, sleep disturbance with medications, intractable diabetes (with a fasting glucose level $>150 \mathrm{mg} / \mathrm{dL}$ or $8.33 \mathrm{mmol} / \mathrm{L}$ or a nonfasting glucose level $>180 \mathrm{mg} / \mathrm{dL}$ or $10.0 \mathrm{mmol} / \mathrm{L}$ ), postvoid residual urine volume $>100 \mathrm{~mL}$, urodynamically proven low-compliance bladder, intractable hypertension (systolic blood pressure $>160 \mathrm{mmHg}$ or diastolic blood pressure $>90 \mathrm{mmHg}$ ), or a previous history of an operation in the pelvic cavity. Biopsyconfirmed men with a prostate-specific antigen (PSA) level $>4.0 \mathrm{ng} / \mathrm{mL}$ were included in the analysis. This trial was performed in accordance with the Declaration of Helsinki, approval for this study was granted by the Institutional Review Board (IRB) of Seoul Metropolitan Government-Seoul National University Boramae Medical Center (IRB No. 16-2014-73), and the content is described according to the STROBE (Strengthening The Reporting of OBservational Studies in Epidemiology) statement.

\section{Study Design}

This was a prospective, multicenter, observational, single-arm 
study and the patients were enrolled at 4 institutions. In accordance with previous investigations [9-14], the treatment response rate in terms of the decrease in nocturnal voids was estimated to be $20 \%$. The standard deviation of the mean response rate was 0.05 and the confidence interval (CI) was $95 \%$. Therefore, the actual number of patients included was calculated to be $65(61 /[1-0.05]=64.2)$, with an anticipated drop-out rate of 5\%. Tamsulosin was supplied by Astellas Pharma (Tokyo, Japan).

Patients were asked whether their nocturia was bothersome at visit 0 . Patients were prescribed $0.2 \mathrm{mg}$ of tamsulosin according to the results, and physicians assessed whether the patients could perform convenience voiding. Patients were advised to take tamsulosin 1 hour before bedtime and to empty their bladders before bed every night. They received a brief explanation about fluid restriction in the evening. Physicians recommended an average daily urine output of 1,500 to 2,000 mL. Caffeinated beverages or fluid-containing food intake after dinner was prohibited. The importance of sleep hygiene was also emphasized to the patients. After 1 month, patients were enrolled in this study if they reported 2 or more nightly voids after taking $0.2 \mathrm{mg}$ of tamsulosin and they provided written informed consent at visit 1 . Patients were prescribed $0.4 \mathrm{mg}$ of tamsulosin and followed up every month at visits 2 and 3 to assess nocturnal voiding and nocturia-related bother. If patients no longer wanted to take tamsulosin they dropped out and were again informed of other medications, including desmopressin and anticholinergic agents.

When patients visited for an outpatient consultation on the relief of LUTS and benign prostatic hyperplasia, a basic examination was performed at visit 0 , including a medical history, physical exam, laboratory tests, PSA testing, urinalysis with microscopy, uroflowmetry with postvoid residual urine, and prostate ultrasonography. At each visit, 3-day frequency-volume charts were obtained and patients responded to questionnaires such as the IPSS, Overactive Bladder Symptom Score (OABSS), International Consultation on Incontinence Questionnaire-Nocturia (ICIQ-N), and nocturia quality of life (N-QoL). Patients' perception of treatment benefit (РPTB), and global response assessment (GRA) at visits 2 and 3 were analyzed after the patients were prescribed $0.4 \mathrm{mg}$ of tamsulosin for 1 or 2 months. The GRA scale was 1 (markedly worse), 2 (moderately worse), 3 (slightly worse), 4 (no change), 5 (slightly improved), 6 (moderately improved), and 7 (markedly improved). These questionnaires were linguistically validated in previous investigations. The 3-day frequency-volume chart was obtained to check the amount of 24-hour urine production, time and volume of day- time and nocturnal voids, bedtime, and time of rising.

\section{Study Endpoints}

The primary endpoint was the change in the mean number of nocturnal voids after 2 months of tamsulosin medication. The secondary parameters were the proportion of $50 \%$ responders from baseline during 2 months of taking tamsulosin, changes in 3-day frequency-volume chart parameters, and changes in scores on the IPSS, OABSS, ICIQ-N, N-QoL, PPTB, and GRA questionnaires. A $50 \%$ response was defined as a decrease in the mean value of nocturnal voids of $>50 \%$ from baseline during 2 months of taking the medication. The variables of the 3-day frequency-volume chart were nocturnal urine volume, maximal bladder capacity, the nocturia index, actual number of nightly voids, the nocturnal polyuria index, and the nocturnal bladder capacity index.

\section{Safety and Tolerability}

Prior to the tamsulosin prescription, a safety assessment was performed that included vital signs, a physical examination, body weight, and the occurrence of any adverse event. All adverse events were recorded at every visit.

\section{Statistical Analysis}

Descriptive data on patients' characteristics, laboratory results, and clinical parameters were described. The paired t-test or Wilcoxon signed-rank test was performed to determine the significance of differences in the number of daytime or nocturnal voids and each parameter of the questionnaires at each followup visit compared to baseline. The Wilcoxon signed-rank test was used when the data did not show a normal distribution or there were $<20$ cases. Multivariate logistic regression analysis was performed to identify predictors of meaningful improvement in nocturia in response to $0.4 \mathrm{mg}$ of tamsulosin. P-values $<0.05$ were considered to indicate statistical significance. All statistical analyses were performed using IBM SPSS Statistics ver. 20.0 (IBM Co., Armonk, NY, USA).

\section{RESULTS}

\section{Patient Characteristics}

In total, 64 male patients were screened at 4 centers, and 2 patients did not satisfy the inclusion criteria (elevated serum creatinine of $1.5 \mathrm{mg} / \mathrm{dL}$ ) and therefore did not receive follow-up as part of the study (Fig. 1). Therefore, 62 patients in the intention- 


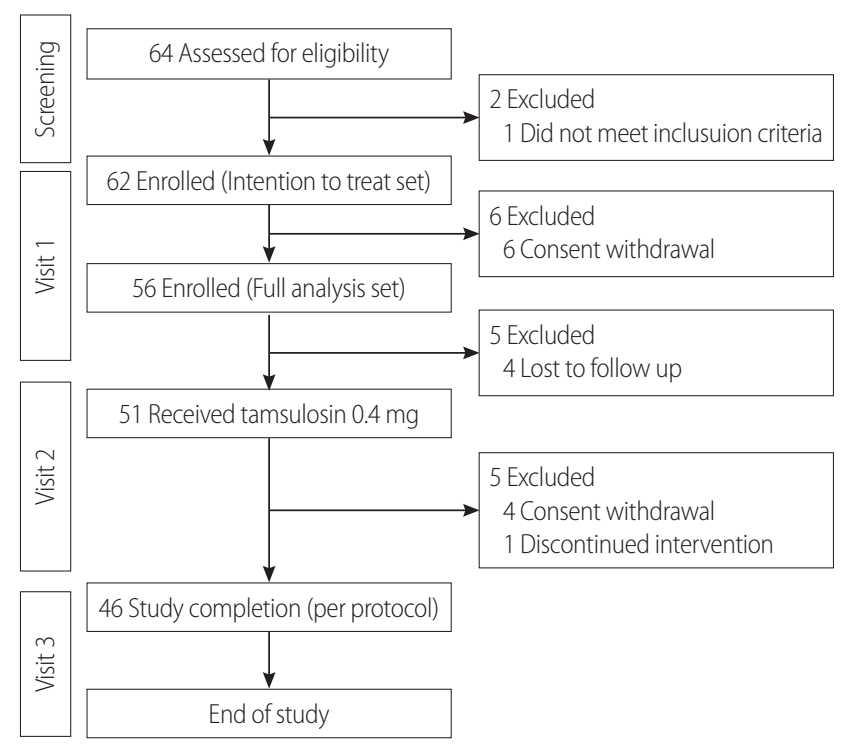

Fig. 1. Study diagram. to-treat (ITT) group were prescribed $0.2 \mathrm{mg}$ of tamsulosin and reported 2 or more nightly voids at visit 0 . Fifty-six of these 62 patients were prescribed $0.4 \mathrm{mg}$ of tamsulosin and were enrolled in the study as the full analysis set (FAS) when they showed intractable nocturia after taking $0.2 \mathrm{mg}$ of tamsulosin for 1 month at visit 1 . Ten patients dropped out at visits 2 and 3 , and 46 patients completed the study. A single case of clinically insignificant orthostatic hypotension was reported as an adverse event.

\section{Primary and Secondary Endpoints}

The patients' mean age was 68 years and there were no statistically significant differences in any demographic parameters or symptom scores between the ITT and FAS groups (Table 1). All parameters except for nocturnal urine volume showed significant improvements at visits 1, 2, and 3 compared to baseline after the patients were enrolled in the study and were prescribed

Table 1. Patient demographics and baseline characteristics

\begin{tabular}{|c|c|c|}
\hline Variable & ITT population $(n=62)$ & FAS population $(n=46)$ \\
\hline Age (yr) & $68.44 \pm 8.73$ & $68.02 \pm 8.69$ \\
\hline Height $(\mathrm{cm})$ & $167.95 \pm 5.99$ & $168.51 \pm 5.82$ \\
\hline Body weight (kg) & $66.78 \pm 7.28$ & $66.73 \pm 7.25$ \\
\hline $\begin{array}{l}\text { Comorbidity } \\
\text { Hypertension } \\
\text { Diabetes mellitus } \\
\text { Sleep disorder } \\
\text { Diabetes insipidus } \\
\text { Previous prostate surgery } \\
\text { Urinary tract infection }\end{array}$ & $\begin{array}{l}31(50.0) \\
16(25.8) \\
4(6.5) \\
0(0) \\
5(8.1) \\
0(0)\end{array}$ & $\begin{array}{l}23(50.0) \\
11(23.9) \\
3(6.5) \\
0(0) \\
2(4.3) \\
0(0)\end{array}$ \\
\hline Glomerular filtration rate $\left(\mathrm{mL} / \mathrm{min} / 1.73 \mathrm{~m}^{2}\right)$ & $80.29 \pm 18.08$ & $81.81 \pm 19.20$ \\
\hline Serum creatinine (mg/dL) & $0.92 \pm 0.15$ & $0.91 \pm 0.16$ \\
\hline Total prostate volume $(\mathrm{mL})$ & $27.43 \pm 10.72$ & $28.4 \pm 12.03$ \\
\hline Transitional prostate volume (mL) & $11.32 \pm 8.59$ & $11.94 \pm 9.96$ \\
\hline $\begin{array}{l}\text { Voiding diary parameters } \\
24 \text {-Hr total urine volume }(\mathrm{mL}) \\
\text { Nocturnal urine volume }(\mathrm{mL}) \\
\text { Total number of nightly voids for } 3 \text { days }\end{array}$ & $\begin{array}{c}1,774.71 \pm 536.70 \\
701.54 \pm 316.06 \\
10.98 \pm 2.06\end{array}$ & $\begin{array}{c}1,835.38 \pm 584.76 \\
711.38 \pm 365.22 \\
11.19 \pm 2.21\end{array}$ \\
\hline $\begin{array}{l}\text { OABSS questionnaires } \\
\text { Total } \\
\text { Question } 2 \text { (urgency) }\end{array}$ & $\begin{array}{r}6.5 \pm 2.52 \\
2.81 \pm 0.40\end{array}$ & $\begin{array}{l}6.23 \pm 2.65 \\
2.82 \pm 0.39\end{array}$ \\
\hline $\begin{array}{l}\text { IPSS questionnaires } \\
\text { Total } \\
\text { Nocturia }\end{array}$ & $\begin{array}{r}20.12 \pm 7.22 \\
3.39 \pm 0.97\end{array}$ & $\begin{array}{r}19.43 \pm 8.05 \\
3.22 \pm 0.85\end{array}$ \\
\hline N-QoL questionnaires & $28.06 \pm 8.70$ & $28.45 \pm 9.19$ \\
\hline
\end{tabular}

Values are presented as mean \pm standard deviation or number (\%).

ITT, intention-to-treat; FAS, full analysis set; OABSS, Overactive Bladder Symptom Score; IPSS, International Prostate Symptom Score; N-QoL, nocturia quality of life. 
Table 2. Change of parameters at follow-up visits compared to baseline

\begin{tabular}{|c|c|c|c|c|}
\hline \multirow{2}{*}{ Variable } & \multirow{2}{*}{ Visits } & \multirow{2}{*}{ Mean \pm SD } & \multicolumn{2}{|c|}{$\mathrm{P}$-value } \\
\hline & & & V0 (baseline) & V1 (baseline) \\
\hline \multirow[t]{4}{*}{ No. of nightly voids } & V0 & $2.71 \pm 0.92$ & Baseline & - \\
\hline & V1 & $2.60 \pm 0.88$ & 0.198 & Baseline \\
\hline & $\mathrm{V} 2$ & $2.08 \pm 0.95$ & $0.285^{\mathrm{a})}$ & $<0.001^{\star}$ \\
\hline & V3 & $1.98 \pm 0.98$ & $0.008^{\mathrm{a})}$ & $<0.001^{\star, \mathrm{a})}$ \\
\hline \multirow[t]{4}{*}{ IPSS total score } & V0 & $20.12 \pm 7.22$ & Baseline & - \\
\hline & V1 & $18.16 \pm 7.19$ & $0.097^{\mathrm{a}}$ & Baseline \\
\hline & $\mathrm{V} 2$ & $15.2 \pm 7.79$ & $0.002^{\star, a)}$ & $<0.001^{\star, a)}$ \\
\hline & V3 & $13.40 \pm 7.09$ & $0.001^{\star, a)}$ & $<0.001^{\star, a)}$ \\
\hline \multirow[t]{4}{*}{ IPSS nocturia score } & V0 & $3.40 \pm 0.97$ & Baseline & - \\
\hline & V1 & $3.10 \pm 1.00$ & $0.034^{\star, \mathrm{a})}$ & Baseline \\
\hline & $\mathrm{V} 2$ & $2.61 \pm 1.04$ & $<0.001^{\star, \mathrm{a})}$ & $<0.001^{\star}$ \\
\hline & V3 & $2.40 \pm 1.08$ & $<0.001^{\star, \mathrm{a})}$ & $<0.001^{\star}$ \\
\hline \multirow[t]{4}{*}{ OABSS total score } & V0 & $6.5 \pm 2.53$ & Baseline & - \\
\hline & V1 & $6.26 \pm 2.27$ & $0.562^{\mathrm{a})}$ & Baseline \\
\hline & $\mathrm{V} 2$ & $5.14 \pm 2.82$ & $0.051^{\mathrm{a})}$ & $0.002^{\star, \mathrm{a})}$ \\
\hline & V3 & $4.74 \pm 2.71$ & $0.003^{*}$ & $<0.001^{\star}$ \\
\hline \multirow[t]{4}{*}{ OABSS urgency score } & V0 & $2.81 \pm 0.40$ & Baseline & - \\
\hline & V1 & $2.61 \pm 0.52$ & $0.013^{\star, \mathrm{a})}$ & Baseline \\
\hline & $\mathrm{V} 2$ & $2.32 \pm 0.77$ & $0.012^{\star, \mathrm{a})}$ & $0.008^{\star, \mathrm{a})}$ \\
\hline & V3 & $2.11 \pm 0.86$ & $<0.001^{\star, \mathrm{a})}$ & $<0.001^{\star, \mathrm{a})}$ \\
\hline \multirow[t]{4}{*}{ Nocturnal urine volume } & V0 & $701.54 \pm 316.06$ & Baseline & - \\
\hline & V1 & $667.14 \pm 231.65$ & 0.987 & Baseline \\
\hline & $\mathrm{V} 2$ & $602.34 \pm 228.02$ & $0.410^{\mathrm{a})}$ & $0.044^{\star}$ \\
\hline & V3 & $631.70 \pm 264.30$ & $0.808^{\mathrm{a})}$ & 0.31 \\
\hline \multirow[t]{4}{*}{ ICIQ-N bothersomeness } & V0 & $8.16 \pm 2.19$ & Baseline & - \\
\hline & V1 & $7.13 \pm 2.54$ & $0.063^{\text {a) }}$ & Baseline \\
\hline & $\mathrm{V} 2$ & $5.74 \pm 3.22$ & $0.025^{\star, \mathrm{a})}$ & $0.003^{\star, \mathrm{a})}$ \\
\hline & V3 & $5.36 \pm 3.17$ & $0.025^{\star, a)}$ & $<0.001^{\star, \mathrm{a})}$ \\
\hline
\end{tabular}

V0, no enrollment with prescription of tamsulosin $0.2 \mathrm{mg}$; V1, patient enrollment with prescription of tamsulosin $0.4 \mathrm{mg}$; V2, visit after 1-month medication of tamsulosin $0.4 \mathrm{mg}$; V3, after 2 months medication of tamsulosin $0.4 \mathrm{mg}$.

SD, standard deviation; IPSS, International Prostate Symptom Score; OABSS, Overactive Bladder Symptom Score; ICIQ-N, International Consultation on Incontinence Questionnaire-Nocturia.

${ }^{\star} \mathrm{P}<0.05,{ }^{\text {a) }}$ Wilcoxon signed-rank test.

Table 3. Multiple logistic linear regression analysis

\begin{tabular}{|c|c|c|c|c|}
\hline Variable & OR & SE & P-value & $95 \% \mathrm{CI}$ \\
\hline Age & 0.91 & 0.09 & 0.354 & $0.755-1.106$ \\
\hline Creatinine & 37.57 & 226.32 & 0.547 & $0-5.04 \times 10^{6}$ \\
\hline Na level & 0.41 & 0.17 & $0.037^{*}$ & $0.178-0.946$ \\
\hline Klevel & 2.24 & 3.61 & 0.615 & $0.096-52.325$ \\
\hline Nocturnal urine volume & 0.99 & 0.01 & $0.025^{\star}$ & $0.978-0.998$ \\
\hline No. of voids & 1.01 & 0.01 & $0.024^{*}$ & $1.002-1.022$ \\
\hline Maximal bladder capacity & 1.01 & 0.01 & 0.103 & $0.998-1.027$ \\
\hline OABSS (Total) & 0.05 & 0.08 & 0.065 & $0.002-1.206$ \\
\hline OABSS (urge incontinence) & 7.08 & 6.61 & $0.036^{*}$ & $1.135-44.163$ \\
\hline
\end{tabular}

OR, odds ratio; SE, standard error; CI, confidence interval; OABSS, Overactive Bladder Symptom Score.

${ }^{\star} \mathrm{P}<0.05$. 
$0.4 \mathrm{mg}$ of tamsulosin for 1 or 2 months. No patients showed $>50 \%$ improvement of nocturia at visit 1 compared to visit 0 . After being prescribed $0.4 \mathrm{mg}$ of tamsulosin, the proportions of the patients with a $>50 \%$ reduction of nocturia were $23.9 \%$ (11 of 46 ) and $22.7 \%$ (10 of 44) in the FAS group at visits 2 and 3, respectively (Table 2 ).

Treatment satisfaction of PPTB was rated as "not effective," "slightly effective," and "very effective" by 12 (19.4\%), 29 (46.8\%), and 10 patients (16.1\%) at visit 2 and by 9 (14.5\%), 29 (46.8\%), and 9 patients (19.4\%) at visit 3, respectively. The GRA scores were $4.92 \pm 1.15$ at visit 2 and $5.11 \pm 1.05$ at visit 3 .

\section{Predictors of the Effective Treatment Group}

Multivariate logistic regression analysis showed that a low serum sodium level (odds ratio [OR], 0.41; 95\% CI, 0.178-0.946; $\mathrm{P}=0.037$ ), nocturnal urine volume (OR, 0.99; 95\% CI, 0.9780.998 ; $\mathrm{P}=0.025$ ), number of voids (OR, 1.01 ; 95\% CI, $1.002-$ $1.022 ; \mathrm{P}=1.01)$, and the OABSS score of urge incontinence $(\mathrm{OR}$, 7.08; 95\% CI, 1.135-44.163; $\mathrm{P}=0.036)$ were significant predictors of $0.4 \mathrm{mg}$ of tamsulosin having a significant improvement of nocturia that was refractory to $0.2 \mathrm{mg}$ of tamsulosin (Table 3 ).

\section{DISCUSSION}

Previous studies have reported the efficacy of low-dose (0.2 mg) tamsulosin for patients with nocturia in Asian countries. Previous studies of the efficacy of $5 \mathrm{mg}$ of terazosin reported 25\%$39 \%$ efficacy for nocturia and improvement of nocturia by $>50 \%$ in $17 \%$ of patients [15]. The MTOPS (Medical Therapy of Prostatic Symptoms) study reported statistically significant improvements of nocturia by 0.77 times in a group of patients receiving $4 \mathrm{mg}$ of doxazosin and by 0.80 times in a combination group of doxazosin and finasteride, compared to improvement of nocturia by 0.61 times in the placebo group. However, this difference seems to be clinically insignificant [16]. Another study investigating the efficacy of $10 \mathrm{mg}$ of alfuzosin showed significant improvement of nocturia by 0.3 times compared to the placebo group [17]. A limitation of these studies is that they used the IPSS questionnaire to evaluate nocturia instead of using a voiding diary, as a voiding diary provides more information regarding patients' nocturia status than the IPSS questionnaire. An additional limitation of these studies is that they did not evaluate other factors, such as patients' prostate size, the improvement of LUTS with concomitant nocturia, treatment satisfaction, and quality of sleep.
In the results of a previous study regarding the administration of $0.2 \mathrm{mg}$ of tamsulosin, Yoshida et al. [12] reported that the patients showed improvements in nocturia and nocturnal polyuria. In particular, in the responder group, it was reported that nocturia had decreased by (on average) 1.4 times, nocturnal urine volume had decreased, and the undisturbed sleep period was prolonged. Two mechanisms were proposed to explain the efficacy of tamsulosin for improving nocturia. The first mechanism was urethral/prostatic alpha-1 receptor inhibition, which alleviated the functional obstruction in the urethra. The second mechanism was the inhibition of urethral sensory nerve activation via reduction of urethral resistance, which resulted in improvements in bladder compliance, storage symptoms, and quality of sleep. These mechanisms may also improve the circadian rhythm of arginine vasopressin and nocturnal polyuria. This possibility was supported by another previous study that demonstrated a possible role of alpha-1A and alpha-1D receptors as the main pathway of vasoconstriction of the renal artery $[18,19]$. A recent study by Kojima et al. [9] showed that the administration of $0.2 \mathrm{mg}$ of tamsulosin, which is a selective alpha$1 \mathrm{~A}$ inhibitor, in the morning, maintained its efficacy on nocturia and nocturnal polyuria for 2 years. However, another study by Yoshimura et al. [20] on efficacy of $0.2 \mathrm{mg}$ of tamsulosin for nocturia reported only a $17.9 \%$ improvement in nocturia (on average), which was the least improvement shown by any symptom in the IPSS questionnaire. Yoshida et al. [12] also reported that nocturia at night only improved by 0.7 times. Furthermore, nocturnal urine volume did not improve in $39.3 \%$ of patients with nocturia that did not respond to $0.2 \mathrm{mg}$ of tamsulosin. The results of the current study were similar, in that nocturnal urine volume only showed a statistically significant improvement at visit 2 compared to baseline. Furthermore, no improvement was found at visit 3 . This may have been because the patients with nocturia did not change their fluid intake patterns.

Logistic regression analysis showed that patients with urge incontinence were 7 times more likely to report improvement in nocturia than those without urge incontinence. This may also have been because severe LUTS, including concomitant urge incontinence and nocturia, can be improved by increasing the dose of tamsulosin. This result is consistent with that of a previous investigation, in which Tyagi et al. [21] reported an association between urge incontinence and nocturnal polyuria in older women. Further studies should be conducted into whether interventions to reduce nocturnal polyuria are associated with the duration of uninterrupted sleep and urge incontinence. 


\section{Limitations}

First, this was a single-arm, observational study that did not compare the efficacy of tamsulosin dose escalation with placebo or another drug for nocturia. However, the authors compared patients who reported that the treatment was "very effective" to those who reported lower levels of satisfaction, which may be helpful when considering tamsulosin dose escalation in patients with nocturia not responding to $0.2 \mathrm{mg}$ of tamsulosin. Second, it is unclear why patients with lower levels of sodium were more likely to experience improvements in nocturia than others. Although many investigators have already recognized that hyponatremia-related symptoms are common in elderly patients, especially those who are hospitalized or living in long-term care facilities [22], the patients enrolled in this study did not show hyponatremic symptoms at the beginning of this study. Further investigation is needed to clarify the effect of sodium levels on nocturia, although tamsulosin dose escalation seems to be safer than using antidiuretic hormone agents for patients with low levels of sodium.

In conclusion, dose escalation to $0.4 \mathrm{mg}$ of tamsulosin yielded overall improvements in terms of the number of nightly voids, IPSS scores, OABSS scores, and ICIQ-N scores. However, it did not show an additional effect on reducing nocturnal urine volume in patients with nocturia compared to $0.2 \mathrm{mg}$ of tamsulosin. Dose escalation may result in significant improvements in nocturia in $>20 \%$ of patients and may be especially helpful for patients with lower levels of sodium and urge incontinence.

\section{AUTHOR CONTRIBUTION STATEMENT}

- Full access to all the data in the study and takes responsibility for the integrity of the data and the accuracy of the data analysis: SYC, HYS

- Study concept and design: SYC

- Acquisition of data: HSY, JWL, JY, MCC, SYC

- Analysis and interpretation of data: SYC, HSY

- Drafting of the manuscript: SYC, HSY

- Critical revision of the manuscript for important intellectual content: SYC, HSY

- Statistical analysis: SYC, HSY

- Obtained funding: SYC, HSY, JWL, MCC

- Administrative, technical, or material support: HSY, JWL, JY, MCC, SYC

- Study supervision: HSY, JWL, JY, MCC, SYC

\section{REFERENCES}

1. Choo MS, Ku JH, Park CH, Lee YS, Lee KS, Lee JG, et al. Prevalence of nocturia in a Korean population aged 40 to 89 years. Neurourol Urodyn 2008;27:60-4.

2. Weiss JP, Blaivas JG. Nocturia. J Urol 2000;163:5-12.

3. Kang DI, Kim HM, Oh SY, Yoon JH, Kim HM, Min KS. Short term effect and safety of antidiuretic hormone in the patients with nocturia. Int Neurourol J 2010;14:227-31.

4. Wang CJ, Lin YN, Huang SW, Chang CH. Low dose oral desmopressin for nocturnal polyuria in patients with benign prostatic hyperplasia: a double-blind, placebo controlled, randomized study. J Urol 2011;185:219-23.

5. Park CH, Chang HS, Oh BR, Kim HJ, Sul CK, Chung SK, et al. Efficacy of low-dose tamsulosin on lower urinary tract symptoms suggestive of benign prostatic hyperplasia : a nonblind multicentre korean study. Clin Drug Investig 2004;24:41-7.

6. Kim SJ, Shin IS, Eun SJ, Whangbo TK, Kim JW, Cho YS, et al. Evidence is enough?: A systematic review and network meta-analysis of the efficacy of tamsulosin $0.2 \mathrm{mg}$ and tamsulosin $0.4 \mathrm{mg}$ as an initial therapeutic dose in asian benign prostatic hyperplasia patients. Int Neurourol J 2017;21:29-37.

7. Bae WJ, Bae JH, Kim SW, Chung BH, Kim JH, Kim CS, et al. Desmopressin add-on therapy for refractory nocturia in men receiving a-blockers for lower urinary tract symptoms. J Urol 2013;190:1806.

8. Cha WH, Choi JD, Kim KH, Seo YJ, Lee K. Comparison and efficacy of low-dose and standard-dose tamsulosin and alfuzosin in medical expulsive therapy for lower ureteral calculi: prospective, randomized, comparative study. Korean J Urol 2012;53:349-54.

9. Kojima Y, Sasaki S, Imura M, Kubota Y, Hayashi Y, Kohri K. Tamsulosin reduces nighttime urine production in benign prostatic hyperplasia patients with nocturnal polyuria: a prospective open-label long-term study using frequency-volume chart. Neurourol Urodyn 2012;31:80-5.

10. Lee HS, Kim SW, Oh SJ, Choo MS, Lee KS. Efficacy and safety of tamsulosin for treating lower urinary tract symptoms associated with benign prostatic hyperplasia: a multicenter, randomized, controlled, open-label non-inferiority study. Korean J Urol 2012;53: 178-83.

11. Zhang K, Yu W, Jin J, Ye H, Wang X, Zhang N, et al. Effect of doxazosin gastrointestinal therapeutic system $4 \mathrm{mg}$ vs tamsulosin 0.2 mg on nocturia in Chinese men with lower urinary tract symptoms: a prospective, multicenter, randomized, open, parallel study. Urology 2011;78:636-40. 
12. Yoshida M, Inadome A, Masunaga K, Nagata T, Yoshiyasu T. Effectiveness of tamsulosin hydrochloride and its mechanism in improving nocturia associated with lower urinary tract symptoms/ benign prostatic hyperplasia. Neurourol Urodyn 2010;29:1276-81.

13. Gorgel SN, Sefik E, Kose O, Olgunelma V, Sahin E. The effect of combined therapy with tamsulosin hydrochloride and meloxicam in patients with benign prostatic hyperplasia symptoms and impact on nocturia and sleep quality. Int Braz J Urol 2013;39:657-62.

14. Simaioforidis V, Papatsoris AG, Chrisofos M, Chrisafis M, Koritsiadis S, Deliveliotis C. Tamsulosin versus transurethral resection of the prostate: effect on nocturia as a result of benign prostatic hyperplasia. Int J Urol 2011;18:243-8.

15. Paick JS, Yang JH, Kim SW, Ku JH. The role of prolactin levels in the sexual activity of married men with erectile dysfunction. BJU Int 2006;98:1269-73.

16. Johnson TM 2nd, Burrows PK, Kusek JW, Nyberg LM, Tenover JL, Lepor $\mathrm{H}$, et al. The effect of doxazosin, finasteride and combination therapy on nocturia in men with benign prostatic hyperplasia. J Urol 2007;178:2045-50.

17. Roehrborn CG, Van Kerrebroeck P, Nordling J. Safety and efficacy of alfuzosin $10 \mathrm{mg}$ once-daily in the treatment of lower urinary tract symptoms and clinical benign prostatic hyperplasia: a pooled analysis of three double-blind, placebo-controlled studies. BJU Int 2003;92:257-61.

18. Villalobos-Molina R, López-Guerrero JJ, Ibarra M. Alpha 1D- and alpha 1A-adrenoceptors mediate contraction in rat renal artery. Eur J Pharmacol 1997;322:225-7.

19. Armenia A, Sattar MA, Abdullah NA, Khan MA, Johns EJ. Functional subtypes of renal alphal-adrenoceptor in diabetic and nondiabetic 2K1C Goldblatt renovascular hypertension. Acta Pharmacol Sin 2008;29:564-72.

20. Yoshimura K, Ohara H, Ichioka K, Terada N, Matsui Y, Terai A, et al. Nocturia and benign prostatic hyperplasia. Urology 2003;61: 786-90.

21. Tyagi S, Perera S, Clarkson BD, Tadic SD, Resnick NM. Nocturnal polyuria in older women with urge urinary incontinence: role of sleep quality, time in bed and medications used. J Urol 2017;197(3 Pt 1):753-8.

22. Filippatos TD, Makri A, Elisaf MS, Liamis G. Hyponatremia in the elderly: challenges and solutions. Clin Interv Aging 2017;12:195765. 